|| ISSN(online): 2589-8698 || ISSN(print): 2589-868X ||

International Journal of Medical and Biomedical Studies

Available Online at www.ijmbs.info

NLM (National Library of Medicine ID: 101738825)

Index Copernicus Value 2019: 79.34

Volume 5, Issue 1; January: 2021; Page No. 301-304

\title{
A QUANTITATIVE, CROSS-SECTIONAL STUDY TO MEASURE AORTA SACRAL PROMONTORY DISTANCE AMONG FEMALES REFERRED FOR CONTRAST ENHANCED CT ABDOMEN
}

\author{
Dr. Hit Narayan Prasad ${ }^{1}$, Dr. Swati Sinha ${ }^{2}$ \\ ${ }^{1}$ Senior Resident, Department of Radiology, Indira Gandhi Institute of Medical Science, Patna, Bihar, India. \\ ${ }^{2}$ Department of Obstetrics and Gynecology, Kurji Holy Family Hospital, Patna, Bihar, India.
}

Article Info: Received 04 January 2021; Accepted 30 January 2021

DOI: https://doi.org/10.32553/ijmbs.v5i1.1709

Corresponding author: Dr. Swati Sinha

Conflict of interest: No conflict of interest.

\begin{abstract}
Aim: This study was carried out to measure the aorta sacral promontory distance among the females referred for contrast enhanced CT abdomen in a tertiary hospital.

Material and methods: A quantitative, cross-sectional study was conducted in the Department of radiology, Indira Gandhi Institute of Medical Science, Patna, Bihar, India from December 2019 to October 2020.Total of 140 patients who underwent Contrast Enhanced CT abdomen. The age, height and weight of the patients were noted. In this study, aorta sacral promontory distance was determined.

Results: The mean age was found to be 51.37 years, mean APT distance $4.61 \pm 0.80 \mathrm{~cm}$ and mean BMI 24.88. Increased in age was correlated with decrease in APT distance but there was no correlation between BMI and APT distance. According to this study, APT distance was decreased in elderly female patients but no significant change in BMI and APT. Conclusion: we conclude that the age increased, APT distance decreased and there was no significant change in relation to BMI. Therefore, during sacral colpopexy, the surgeon should be careful and consider the aorta sacral promontory distance in the elder female patients while performing dissection.
\end{abstract}

Keywords: aorta sacral promontory distance, pelvic organ prolapse, sacral colpopexy.

\section{Introduction}

All the pelvic organs are supported by complex "hammock" muscles, ligaments and fibers that attach to the bony anatomy of the pelvis. When these are weakened those organ can drop. A prolapse happens when the pelvis muscles and tissues can no longer support these organs because the muscles and tissues are weak or damaged. This causes one or more pelvic organs to drop or press into or out of the vagina. Pelvic organ prolapse is a downward descent of female pelvic organs, including the bladder, uterus and the small or large bowel, resulting in protrusion of the vagina, uterus or both. Prolapse development can be attributed to several factors, including vaginal child birth, advancing age and obesity. Pelvic organ prolapse disorder are more common in elderly female population. Women who had vaginal delivery have a higher incidence of pelvic organ prolapse than women who have undergone caesarean delivery. ${ }^{1}$ Sacral colpopexy has been consistently shown to correct vaginal vault prolapse with an anatomical success rate of 78-100\% and a subjective cure rate as high as 92.1 $\% .^{2}$ However, rare but serious intraoperative complications of hemorrhage may be encountered. The need for blood transfusion occurs at a rate of $4.4 \% .^{1}$ There is the potential for vascular and organ injuries, some of which may not be recognized until postoperatively. ${ }^{1,}, 3,4$ To avoid such complications, the surgeon should be aware of the proximity of nearby vessels such as the common iliac veins, iliac arteries, and the aortic bifurcation. ${ }^{3}$ The location of the aortic bifurcation and sacral promontory provides anatomical orientation during complex surgical dissection. It is crucial for the surgeon to be cognizant of the potential anatomical variability in patients. In the United States pelvic floor disorders affect almost $25 \%$ of the female population. These disorders are more prevalent in the elderly population. ${ }^{5}$ As more patients are seeking definitive management for their prolapse, the number of elderly patients undergoing sacral colpopexies will likely increase. Some studies have demonstrated that with increasing age, the aorta enlarges and elongates. ${ }^{6}$ Elderly patients also suffer from diseases that affect the spinal column, such as osteoarthritis, osteoporosis, or degenerative disc disease, which may alter the height of the vertebral bodies. ${ }^{6}$ Sacral colpopexy is a surgical technique for repairing pelvic organ prolapse in women. A higher BMI have been associated with increased prevalence of pelvic floor disorders. ${ }^{7}$ Some studies have demonstrated that with increase in age, aorta enlarges and elongates. ${ }^{8,9}$ During sacral colpopexy, likelihood of a decreased APT distance in elderly and hypertensive patients require careful identification of aorta bifurcation and sacral promontory before dissection

Therefore, this study was conducted to aid surgeons who operate for pelvic disorder for careful identification of aorta and sacral promontory before dissection to avoid complication. The specific objectives of this study were to 
estimate the aorta sacral promontory distance in female patients and to correlate aorta sacral promontory distance with age and BMI.

\section{Material and Methods}

A quantitative, cross-sectional study was conducted in the Department of radiology, Indira Gandhi Institute of Medical Science, Patna, Bihar, India from December 2019 to October 2020, after taking the approval of the protocol review committee and institutional ethics committee.

Methodology

Total 140 patients who visited for CECT scan of abdomen without pathological findings were included in the study. Measurement was performed on CECT scan of abdomen on sagittal section. Female patients aged 20 and above referred for CECT scan of abdomen without pathological finding were included in the study. Patients with sacral injury, aortic and spinal diseases were excluded in the study. The protocol parameters under CECT Protocol in TUTH were as $600 \mathrm{ml}$ plain water $30 \mathrm{~min}$ before and then $400 \mathrm{ml}$ just before examination, Scanning area from dome of diaphragm to iliac crest, Detect or collimation:0.6x128,Pitch: $\quad 0.85, \mathrm{mAs}$ : 200,Contrast: Intravenous, Volume: $80-100 \mathrm{ml}$ at rate of $3.5-4 \mathrm{ml} / \mathrm{s}$ and Recon slice and interval:5x5mm with triple phase scan delay as $15-20 \mathrm{sec}$ for arterial phase, 40-60 sec for portal phase and 70-80 sec for venous phase .

In this study, measurement of aorta sacral promontory was measured by reconstructing arterial phase of the abdomen on sagittal image, point of aortic bifurcation was identified on coronal image and then distance from level of aortic bifurcation to sacral promontory was measured. Vertical distance was represented as aorta sacral promontory distance.

Patient's age, weight, height was recorded. Data was obtained and analyzed using the descriptive statistics to summarize the information, and inferential statistics (paired sample t-test) to verify if there were significant difference between patient's APT distance with age and BMI.

\section{Results}

Data was collected from 140 Patients who underwent CECT scan for abdomen. Female patients were categorized into four age groups of 20-40, 40-60, 60-80, and above 80 . The mean age was 52.33 years (range $21-85$ years). Total patients in age groups of 20-40 years was $36.42 \%$, 40-60 years was $34.28 \%$, 60-80 years was $22.86 \%$ and in age group of 80 years above, number of patients were $7.85 \%$ (Table 1). As the age group increased, there was a decrease in mean APT distance. The mean APT distance was longer among the age group of 20-40 years and obese group. APT distance was short in elderly female patients and underweight patients. The mean aorta sacral promontory distance of age group 20-40 years was found to be
$5.32 \pm 0.52 \mathrm{~cm}, 40-60$ years to be $5.11 \pm 0.55 \mathrm{~cm}, 60-80$ years to be $3.77 \pm 0.48 \mathrm{~cm}, 80$ years above to be $2.65 \pm 0.37 \mathrm{~cm}$. As the age group increased, there was a decrease in mean APT distance. The mean APT distance was longer among the age group of 20-40 years. Mean BMI was 25.14 (range 17.94-33.21). The number of patients in underweight group $(\mathrm{BMI}<18.5)$ was 15 and mean APT distance was $17.94 \pm 0.52 \mathrm{~cm}$, normal BMI group (18.5-25) was 82 with mean APT distance $22.44 \pm 1.84 \mathrm{~cm}$, in overweight group (25-30) was 40 and mean APT was $27.41 \pm 1.52 \mathrm{~cm}$, in the obese group ( 30 and above) there were 3 patients with mean APT distance $33.21 \pm 3.32 \mathrm{~cm}$. As BMI increased in patients, there was increase in APT distance (Table 3). There was a strong negative correlation between APT and age. Increased in age was correlated with decrease in APT distance (Pearson's correlation coefficient (r) was found0.732 between age and APT distance, $\mathrm{p}<0.01)$. There was no significant correlation between BMI and APT distance (Pearson's correlation coefficient (r) was found-0.011 between BMI and APT distance, p>0.01) (Table 4). Hence, age and BMI were responsible for variation in APT distance. Therefore, there was an overall significant relation between APT distances with age .There was strong negative correlation between APT distance and age (correlation coefficient $\left(\mathrm{r}^{2}\right)$ value was found to be 0.577). Increased in age leads to decrease in APT distance. There was no significant correlation between BMI and APT distance (correlation coefficient $\left(\mathrm{r}^{2}\right)$ value was found to be between BMI and APT distance.

Table 1: Distribution of sample size according to age group

\begin{tabular}{lll}
\hline Age (in years) & Number of patients $=\mathbf{1 4 0}$ & $\%$ \\
\hline $20-40$ & 51 & $36.42 \%$ \\
\hline $40-60$ & 48 & $34.28 \%$ \\
\hline $60-80$ & 30 & $22.86 \%$ \\
\hline Above 80 & 11 & $7.85 \%$ \\
\hline
\end{tabular}

Table 2: Comparison of mean aorta sacral promontory distance with age

\begin{tabular}{ll}
\hline $\begin{array}{l}\text { Age } \\
\text { (in years) }\end{array}$ & $\begin{array}{l}\text { Aorta sacral promontory distance }(\mathbf{c m}) \\
\text { mean } \pm \text { standard deviation }\end{array}$ \\
\hline $20-40$ & $5.32 \pm 0.52$ \\
\hline $40-60$ & $5.11 \pm 0.55$ \\
\hline $60-80$ & $3.77 \pm 0.48$ \\
\hline Above 80 & $2.65 \pm 0.37$ \\
\hline
\end{tabular}

Table 3: Comparison of mean aorta sacral promontory distance with BMI

\begin{tabular}{lll}
\hline BMI & $\begin{array}{l}\text { Number of } \\
\text { patients }\end{array}$ & $\begin{array}{l}\text { Aorta sacral promontory } \\
\text { distance }(\mathbf{c m}) \quad \begin{array}{l}\text { mean } \\
\text { standard deviation }\end{array}\end{array}$ \\
\hline Underweight $(<18.5)$ & 15 & $17.94 \pm 0.52$ \\
\hline Normal $(18.5$ to $<25)$ & 82 & $22.44 \pm 1.84$ \\
\hline Overweight $(25$ to 30$)$ & 40 & $27.41 \pm 1.52$ \\
\hline Obese $(30$ and above) & 3 & $33.21 \pm 3.32$ \\
\hline
\end{tabular}


Table 4: Linear regression model for predictors of APT distance

\begin{tabular}{lll}
\hline Variable & r-value & p-value \\
\hline Age & -0.732 & $<0.01$ \\
\hline Body mass index & -0.011 & $>0.01$ \\
\hline
\end{tabular}

\section{Discussion}

The number of elderly patients undergoing surgeries for pelvic floor disorders is increasing gradually in recent years. ${ }^{1}$ As more patients are seeking surgical treatment for pelvic prolapse, surgeons should be familiar with proximity of nearby vessels to avoid complications to the iliac artery, iliac veins and aortic bifurcation. Before a surgery, it will be beneficial for surgeons if APT distance is measured using CT scans which can prevent intraoperative complications like injury to vessels, haemorrhage, ureteral injury and enterotomy. This study showed that as the age group increases, there was significant decrease in mean APT distance. However, as BMI increased in patients, there was increase in APT distance but was not significant. APT distance was short in elderly female patients. In this study the mean aorta sacral promontory distance of age group 20 40 years was found to be $5.32 \pm 0.52 \mathrm{~cm}, 40-60$ years to be $5.11 \pm 0.55 \mathrm{~cm}, 60-80$ years to be $3.77 \pm 0.48 \mathrm{~cm}, 80$ years above to be $2.65 \pm 0.37 \mathrm{~cm}$. As the age group increased, there was a decrease in mean APT distance. This result agreed with the result of the study conducted by Sneha Mary Varghese, Suresh Sukumar, Abhimanyu Pradhan. ${ }^{10}$ From 172 subjects the mean age was 56.5 years (18-95) and mean BMI was 23.25 (range 10.8-35.7). The mean APT distance was found to be $2.7 \pm 15 \mathrm{~cm}$. The study also showed that as the age group increased, there was a significant decrease in mean APT distance. However, as the BMI increased in patients, there was no significant change in mean APT distance. The observation in this present study was similar with earlier research conducted by Agrawal A et al. ${ }^{8}$ Mean age was 56.6 years and mean APT distance measured on CT was $6.3 \pm 1.02 \mathrm{~cm}$. This study also supported that within the increasing age group, the mean APT distance significantly decreased. With increase in age there was degeneration of intervertebral discs which shortens the vertebral column and thus a lower level of aortic bifurcation may result. ${ }^{11}$ In the study conducted by Forbang NI et al., to find the association between cardiovascular disease and aortoiliac bifurcation in 1711 participants, the bifurcation position was determined using CT by measuring Aorto-Iliac Bifurcation Distance (AIBD). ${ }^{12}$ The mean AIBD was found to be $26 \pm 15 \mathrm{~mm}$ and the mean age was $62 \pm 10$ years. The present study showed similar results with Forbang NI et al., study with the mean distance of $27.96 \pm 0.63 \mathrm{~mm}$ and mean age of $56.5 \pm 8$ years. Also, in Lee $\mathrm{CH}$ et al., and Voboril R et al., study, the results of the common site of aortic bifurcation was at L4 vertebra which was same as present study. ${ }^{13,14}$ Major limitations of this study was large number of patients could not be included. We measured patient's parameter manually and the value may not be consistent. We measured the parameters of different patients so the level of the site of measurement might not be the same in all cases. Due to less time period, the number of female patients in BMI group $>30$ and also number of patients in age group $>80$ years was quite less. This type of study should be done in larger number of normal adult population to make the study more reliable.

\section{Conclusion}

We concluded that the age increased, APT distance decreased and there was no significant change in relation to BMI. Therefore, during sacral colpopexy, the surgeon should be careful and consider the aorta sacral promontory distance in the elder female patients while performing dissection

\section{Reference}

1. Nyaard IE, McCreey R, Brubaker L, Connolly AM, Cundiff G, Weber AM, Zczynski H (2004) Abdominal sacrocolpopexy: a comprehensive review. Obstet Gynecol 104:805-823

2. Barber MD, Brubaker L, Nygaard I, Wheeler TL, Schaffer J, Chen Z, Spino C (2009) Defining success after surgery for pelvic organ prolapse. Obstet Gynecol 114:600-609

3. Good MM, Abele TA, Balgobin S, Montoya I, McIntire D, Corton MM (2013) Vascular and ureteral anatomy relative to the midsacral promontory. Am J Obstet Gynecol 208:486, e1-7

4. Visco AG, Advincula A (2008) Robotic gynecologic surgery. Obstet Gynecol 112:1369-1384

5. Wu JM, Vaughan CP, Goode PS, Redden DT, Burgio KL, Richter HE, Markland AD (2014) Prevalence and trends of symptomatic pelvic floor disorders in US Women. Obstet Gynecol 123:141148

6. Kornreich L, Hadar H, Sulkes J, Gornish M, Ackerman J, Gadoth N (1998) Effect of normal ageing on the sites of aortic bifurcation and inferior vena cava confluence: a CT study. Surg Radiol Anat 20:63-68

7. Hendrix SL, Clark A, Nygaard I, Aragaki A, Barnabei V, McTiernan A. Pelvic organ prolapse in the Women's Health Initiative: Gravity and gravidity. Am J Obstet Gynecol. 2002;186(6):116066.

8. Agrawal A, Abayazeed A, Francis SL, Tolentino J, Ostergard DR, Seow A, et al. Correlation of patient age with CT-measured aorta sacral promontory distance. Int Urogynecol J Pelvic Floor Dysfunct. 2015;26(6):887-91.

9. Kornreich L, Hadar H, Sulkes J, Gornish M, Ackerman J, Gadoth N. Effect of normal ageing on the sites of aortic bifurcation and inferior vena cava 
confluence: a CT study. Surg Radiol Anat [Internet]. Springer-Verlag. 1998;20(1):63-68.

10. Varghese S, Sukumar S, Pradhan A. Comparison of Aorta-sacral Promontory Distance with Age and BMI in Female Patients Undergoing CT. Journal of Clinical \& Diagnostic Research. 2017;11(10).

11. Appaji AC, Kulkarni R, Pai SB. Level of bifurcation of aorta and iliocaval confluence and its clinical relevance. IOSR J Dent Med Sci. 2014;13:56-60.

12. Forbang NI, Ix JH, Allison MA, Criqui MH. Associations of cardiovascular disease risk factors and calcified atherosclerosis with aortoiliac bifurcation position: the multiethnic study of atherosclerosis. Angiology [Internet]. 2013;66(1):90-95.

13. Lee CH, Seo BK, Choi YC, Shin HJ, Park JH, Jeon $\mathrm{HJ}$, et al. Using MRI to evaluate anatomic significance of aortic bifurcation, right renal artery, and conusmedullaris when locating lumbar vertebral segments. Am J Roentgenol. 2004;182(5):1295-300.

14. Voboril R. The position of the aortic bifurcation in humans. Sb Ved Pr Lek Fak Karlovy Univerzity Hradci Kralove Suppl. 1993;36(1-2):87-104 